Article

\title{
Double-Layer E-Structure Equalization Circuit for Series Connected Battery Strings
}

\author{
Shungang Xu, Kai Gao, Xiaobing Zhang * and Kangle Li \\ School of Electrical Engineering, Southwest Jiaotong University, Chengdu 611756, China; \\ Shungang_xu@163.com (S.X.); gaokai_pece@163.com (K.G.); Likangle_pece@163.com (K.L.) \\ * Correspondence: Zhangxb_pece@163.com; Tel.: +86-15283329184
}

Received: 10 October 2019; Accepted: 5 November 2019; Published: 8 November 2019

\begin{abstract}
In order to eliminate the voltage imbalance among battery cells when they are connected in series, the paper proposes a double-layer E-structure (DLE) equalizer based on bidirectional buck-boost converters, which has the advantage of quick equalization speed and can be applied to arbitrary number batteries. Furthermore, a novel two-stage equalization control strategy is proposed for the DLE equalizer to decrease maximum voltage gap between the maximum and minimum voltage cells. The paper analyses the working principle of proposed equalizer in detail and describes the detailed design of the control strategy and implement process. Simulation and experiment results show that the proposed equalizer can improve equalization performance of battery cells compared with adjacent cell-to-cell (AC2C) equalizer.
\end{abstract}

Keywords: series-connected battery string; buck-boost converter; voltage gap; equalizers

\section{Introduction}

Nowadays, lithium-ion batteries are more and more widely used in the field of electric vehicles (EVs). Since EVs require higher voltage than what a single storage cell can output, large number of cells are needed to be connected in series [1,2]. However, cell voltage imbalance among series-connected batteries will occur due to manufacturing and environmental differences among them. This imbalance will lead to the decay of available battery capacity and lifetime of batteries. Therefore, battery equalizers are needed to eliminate the voltage imbalance among battery cells and to improve system performance.

In order to equalize the energy among the mismatch cells connected in series, many battery equalizers have been developed [3,4]. These equalizers can be classified into two categories: passive and active battery equalizers. Passive battery equalizers have the advantages of low cost and small size, because they just need a resistor and a switch for each cell $[5,6]$. However, the energy dissipation of resistor degrades the efficiency and might cause thermal issue. In order to overcome the drawback of the passive equalizers, many active battery equalizers have been proposed. These equalizers transfer excessive energy from the higher voltage cells to the lower voltage cells by using capacitors or inductors. Typical active battery equalizers include switched capacitor equalizers [7-9], equalizers based on bidirectional buck-boost converters [10-16], and transformer equalizers [17-19]. Among them, switched capacitor equalizers have advantages of small volume and simple control strategy, such as single-tiered switched capacitor [7], and improved switched capacitor [8,9], but their equalization speeds are slow. Transformer equalizers have advantages of flexible equalization path and quick equalization speed, such as single-winding transformer equalizer [18], and multi-winding transformer equalizers $[17,19]$, but leakage inductor of transform lead to the appearance of equalization deviation. Equalizers based on the bidirectional buck-boost converters have the advantages of the bidirectional energy flow, easy modularization, and simple structure. Literature [10] proposes an adjacent cell-to-cell (AC2C) equalizer based on bidirectional buck-boost converters. The $\mathrm{AC} 2 \mathrm{C}$ equalizer is featured with 
arbitrary battery number and simple control, but the redundant energy of higher voltage cell can only be transferred between two adjacent cells. When an AC2C equalizer is applied to a large battery string, it has many shortcomings, such as slow equalization speed, low equalization efficiency, and huge voltage gap between maximum and minimum voltage cells in the battery string. Literature [11] improves the equalization speed by exchanging the positions of switches and inductors, but the equalizer control strategy is complicated. Studies $[12,13]$ have improved the equalization speed by changing the connection of equalizer unit, but their cost are increased. Studies $[14,15]$ introduce two methods to improve equalization speed and efficiency, but the huge voltage gap after equalization is still existed. Reference [16] proposes a parallel architecture equalizer based on buck-boost converter. It has fast equalization speed and high equalization efficiency compared to the AC2C equalizer. However, it is worth mentioning that the parallel structure is difficult to extend. In other words, the number of batteries is restricted. Figure 1 shows $\mathrm{AC} 2 \mathrm{C}$ equalizer and parallel structure equalizer, which are commonly used in battery equalization mentioned above. Figure 1a shows the AC2C equalizer. Figure $1 \mathrm{~b}$ shows the parallel architecture equalizer.

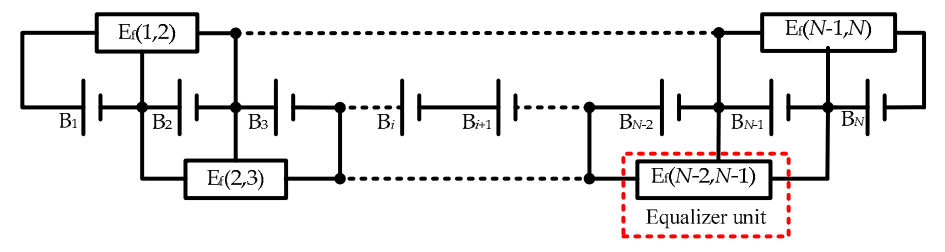

(a)

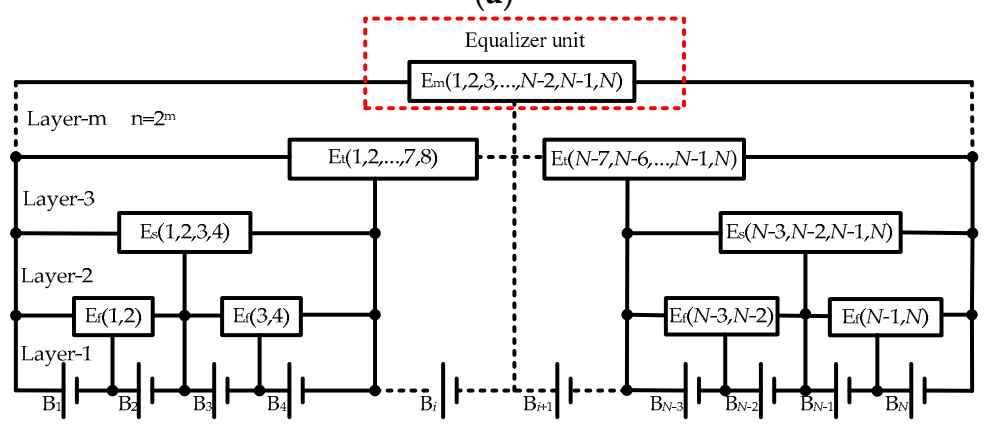

(b)

Figure 1. $\mathrm{AC} 2 \mathrm{C}$ equalizer and parallel structure equalizer. (a) $\mathrm{AC} 2 \mathrm{C}$ equalizer; (b) parallel structure equalizer.

A double-layer E-structure (DLE) battery equalizer is proposed in this paper. The proposed equalizer has the advantage of quick equalization speed and can be applied to arbitrary number batteries. In addition, a novel two-stage control strategy is proposed to decrease the maximum voltage gap after equalization. The structures of proposed equalizer are described in Section 2. The equalization principle is described in Section 3. The equalization control strategy is described in Section 4 . The simulation results are described in Section 5 . The experimental results are described in Section 6, followed by the conclusion in Section 7.

\section{Structures of Proposed Equalizer}

The structures of proposed equalizer are shown in Figure 2. Figure $2 a, b$ show the odd structure and even structure of proposed equalizer, respectively. The proposed equalizer is composed of the inner-layer equalizer units and the outer-layer equalizer units. $\mathrm{E}_{\mathrm{f}}(i, i+1)$ represents the inner-layer equalizer unit and it equalizes the cells $B_{i}$ and $B_{i+1} . E_{s}(j, j+1, j+2, j+3)$ represents outer-layer equalizer unit and it equalizes the cell substrings $\left(\mathrm{B}_{i}, \mathrm{~B}_{i+1}\right)$ and $\left(\mathrm{B}_{i+2}, \mathrm{~B}_{i+3}\right)$.

When cell number $N$ is odd, the inner-layer equalizer units of proposed equalizer include $E_{f}(1,2)$, $\mathrm{E}_{\mathrm{f}}(3,4), \ldots, \mathrm{E}_{\mathrm{f}}(i, i+1), \ldots, \mathrm{E}_{\mathrm{f}}(N-2, N-1), \mathrm{E}_{\mathrm{f}}(N-1, N)$ and its outer-layer equalizer units include 
$\mathrm{E}_{\mathrm{s}}(1,2,3,4), \mathrm{E}_{\mathrm{s}}(3,4,5,6), \ldots, \mathrm{E}_{\mathrm{s}}(j, j+1, j+2, j+3), \ldots, \mathrm{E}_{\mathrm{s}}(N-4, N-3, N-2, N-1)$. The number of equalizer units can be expressed as

$$
\left\{\begin{array}{l}
\text { Inner-layer : } N_{f}=\frac{N+1}{2} \\
\text { Outer-layer }: N_{s}=\frac{N-3}{2}
\end{array}\right.
$$

where $N_{f}$ and $N_{s}$ are the number of inner-layer and outer-layer equalizer units, respectively.

When $N$ is even, the inner-equalizer units of proposed equalizer include $E_{\mathrm{f}}(1,2), \mathrm{E}_{\mathrm{f}}(3,4), \ldots$, $\mathrm{E}_{\mathrm{f}}(i, i+1), \ldots, \mathrm{E}_{\mathrm{f}}(N-1, N)$, and its outer-layer equalizer units include $\mathrm{E}_{s}(1,2,3,4), \mathrm{E}_{s}(3,4,5,6)$, $\ldots, \mathrm{E}_{s}(j, j+1, j+2, j+3), \ldots, \mathrm{E}_{s}(N-3, N-2, N-1, N)$. The number of equalizer units can be expressed as

$$
\left\{\begin{array}{c}
\text { Inner-layer : } N_{f}=\frac{N}{2} \\
\text { Outer-layer : } N_{s}=\frac{N-2}{2}
\end{array}\right.
$$

Each equalizer unit of proposed equalizer is a bidirectional buck-boost converter, as shown in Figure 3. Figure 3a shows an inner-layer equalizer unit that equalizes two adjacent cells; Figure $3 b$ shows an outer-layer equalizer unit that equalizers two adjacent cell substrings. The proposed equalizer is composed of $(2 N-2)$ switches (inner-layer switches: $S_{1}-S_{N}$, outer-layer switches: $\left.S_{N+1}-S_{2 N-2}\right)$ and ( $N$ -1 ) inductors, as many as the traditional $A C 2 C$ equalizer. In addition, similar to the parallel structure equalizer, the proposed equalizer provides flexible equalization paths, which reduces equalization time and improves equalization efficiency.

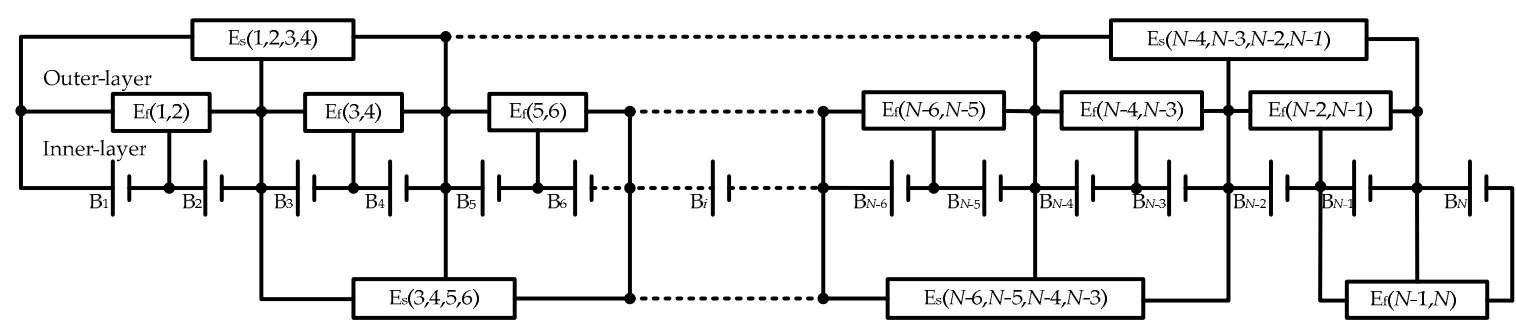

(a)

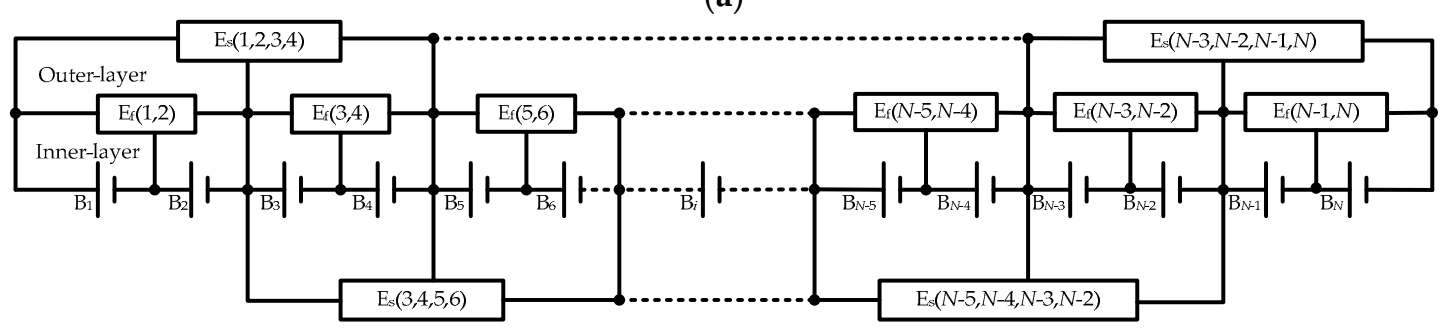

(b)

Figure 2. Structures of proposed equalizer. (a) Odd structure of proposed equalizer. (b) Even structure of proposed equalizer.

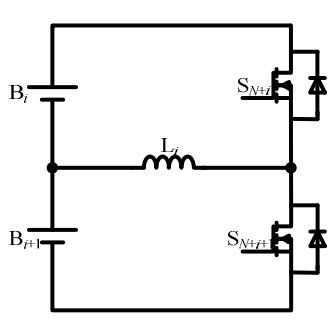

(a)

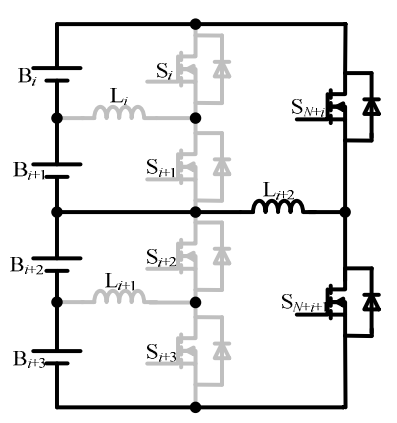

(b)

Figure 3. Equalizer unit. (a) Inner-layer equalizer unit. (b) Outer-layer equalizer unit. 
To facilitate the analysis, several assumptions are made as follows:

1. All diodes, switches, and inductors employed in proposed equalizer are ideal;

2. Each inductor has the identical parameter, i.e., $L=L_{1}=L_{2}=L_{3}=\ldots=L_{2 N-2}$;

3. Cell voltages are constant during one switching period;

4. All of the buck-boost converters are operated in discontinuous conduction mode (DCM)

\section{Equalization Principles}

The equalization process of the proposed equalizer consists of two stages. In the first stage, the energy is transferred among adjacent cell substrings and between two cells in a substring by corresponding outer-layer and inner-layer equalizer units, respectively. It is noted that, when $N$ is odd, the energy can also be transferred between cells $B_{N}$ and $B_{N-1}$, which are adjacent cells but belong to different substrings. In the second stage, the energy is transferred from the maximum voltage cell to the minimum voltage cell, which is implemented by multiple inner-layer and outer-layer equalizer units. By the first stage equalization process, adjacent cell substrings and two cells in a substring are equalized, but the imbalance among cells in the whole battery string is still large. The second stage equalization can realize the equalization of the battery string with small maximum voltage gap among cells, but its equalization speed is slower. Thus, the proposed equalization control strategy combines the first with second stage equalization, which has advantages of both the first and second stage equalization.

\subsection{First Stage Equalization}

In the first stage equalization, the inner-layer equalizer and outer-layer equalizer work independently. The operation of the equalizer is based on the voltage gap between two adjacent cells or cell substrings. The equalizer has two predefined equalization thresholds; one predefined threshold $\Delta V$ for the inner-layer equalizer unit and one predefined threshold $2 \Delta V$ for out-layer equalizer unit. If the voltage gap is less than the predefined equalization threshold, the corresponding equalizer unit stops working. The working condition of inner-layer equalizer unit can be expressed as

$$
\left|V_{\mathrm{B} i}-V_{\mathrm{B} i+1}\right|>\Delta V\left\{\begin{array}{c}
N \text { is odd }: i=1,3,5, \ldots, N-2, \text { and } i=N-1 \\
N \text { is even }: i=1,3,5, \ldots, N-1
\end{array}\right.
$$

where $V_{\mathrm{B} i}$ is the voltage of cell $\mathrm{B}_{i}$. The working condition of outer-layer equalizer unit can be expressed as

$$
\left|\left(V_{\mathrm{B} i}+V_{\mathrm{B} i+1}\right)-\left(V_{\mathrm{B} i+2}+V_{\mathrm{B} i+3}\right)\right|>2 \Delta V\left\{\begin{array}{c}
N \text { is odd }: i=1,3,5, \ldots, N-4 \\
N \text { is even }: i=1,3,5, \ldots, N-3
\end{array}\right.
$$

When both the inner-equalizer unit and outer-layer equalizer unit stop working, the equalization process ends. During this stage, the operation principles of inner-layer and outer-layer equalizer units are similar. For the sake of simplification, take inner-equalizer unit as an example to analyze the working process. Assuming that cell voltage $V_{\mathrm{B} i}$ is higher than cell voltage $V_{\mathrm{B} i+1}$. The equalization process of this stage includes two modes, i.e., Mode 1 and Mode 2. The current paths of the first stage equalization during different modes are shown in Figure 4.

Mode $1\left[t_{0}-t_{1}\right]: \mathrm{B}_{i}$ discharges.

Figure 4a presents the inductor current path of Mode 1 . Mode 1 starts when the switch $S_{i}$ is turned on and the switch $S_{i+1}$ is turned off. Then, $V_{\mathrm{B} i}$ is directly applied to the terminal of the inductor $L_{i}$, and the inductor current $i_{L i}$ is built up. During this mode, $L_{i}$ is charged by cell $\mathrm{B}_{i}$ and $i_{L i}$ increases linearly. Therefore, $i_{L i}$ can be expressed as

$$
i_{L i}=\frac{V_{\mathrm{B} i}}{L_{i}}\left(t-t_{0}\right), t_{0}<t<t_{1}
$$


The energy $W_{i}$ is transferred from cell $\mathrm{B}_{i}$ to $L_{i}$, and it can be expressed as

$$
W_{i}=V_{\mathrm{B} i} \int_{0}^{D_{i} T_{s}}\left(\frac{V_{\mathrm{B} i}}{L_{i}} t\right) d t=\frac{V_{\mathrm{B} i}^{2}}{2 L_{i}} D_{i}^{2} T_{s}^{2}
$$

In the Formula (6), $D_{i}$ is the duty cycle of the switch $S_{i}$, and $T_{\mathrm{s}}$ is the switching period.

Mode $2\left[t_{1}-t_{2}\right]: \mathrm{B}_{i+1}$ charges.

Figure $4 \mathrm{~b}$ presents the inductor current path of Mode 2. Mode 2 begins when the switches $S_{i}$ and $S_{i+1}$ are turned off. The inductor current is commutated from $S_{i}$ to the parasitic diode of $S_{i+1}$, and it decreases linearly due to the cell voltage is applied to the inductor in the opposite direction. During this mode, the energy is transferred into cell $\mathrm{B}_{i+1}$ and $V_{\mathrm{B} i+1}$ increases slowly. $i_{L i}$ can be expressed as

$$
i_{L}=\frac{V_{\mathrm{B} i}}{L}\left(t_{1}-t_{0}\right)-\frac{V_{\mathrm{B} i+1}}{L}\left(t-t_{1}\right), t_{1}<t<t_{2}
$$

When $i_{L}$ reduces to zero, the energy transportation will be stopped by the parasitic diode of $S_{i+1}$. The freewheeling time $T_{d}$ of $i_{L}$ can be expressed as

$$
T_{d}=\frac{V_{\mathrm{B} i}}{V_{\mathrm{B} i+1}}\left(t_{1}-t_{0}\right)
$$

In order to avoid magnetic saturation of inductors, the duty cycle of all switches should be designed to satisfy the following expression

$$
D_{i}=\frac{t_{1}-t_{0}}{T_{s}}<\frac{t_{1}-t_{0}}{T_{d}+t_{1}-t_{0}}=\frac{V_{\mathrm{B} i}}{V_{\mathrm{B} i}+V_{\mathrm{B} i+1}}
$$

The normal voltage range of lithium-ion battery is $2.8-4.2 \mathrm{~V}$, so the duty cycle of all switches must be less than 0.4 to avoid magnetic saturation.

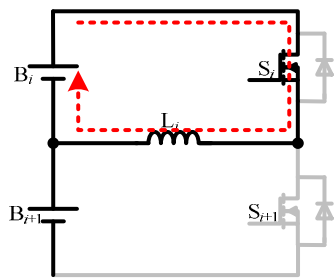

(a)

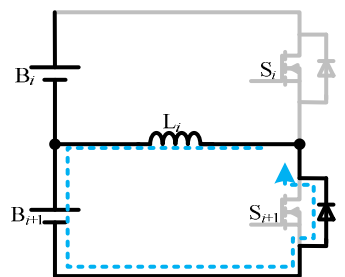

(b)

Figure 4. Current paths of the first stage equalization during different modes. (a) Mode $1, \mathrm{~B}_{i}$ discharges; (b) Mode 2, $\mathrm{B}_{i+1}$ charges.

\subsection{Second Stage Equalization}

The second stage equalization forms an equivalent equalization path by controlling the conduction of the switches and regulating specific duty cycle to achieve equalization between the maximum voltage cell and minimum voltage cell. The second stage equalization includes three modes: Mode 1 , the energy transfers from the maximum voltage cell to outer-layer inductor; Mode 2, the energy flows among outer-layer inductors; Mode 3, the energy transfers from outer-layer inductor to the minimum voltage cell. The paper takes even structure of proposed equalizer as an example to elaborate the operation process of the second stage equalization. It is supposed that the voltage of cell $\mathrm{B}_{i}$ is maximum and the voltage of cell $\mathrm{B}_{j}$ is minimum.

Mode 1: the energy transfers from the maximum voltage cell to outer-layer inductor.

Whether switches work or not in equalization process is related to the position of maximum voltage cell $\mathrm{B}_{i}$ in Mode 1, and the corresponding relationship is shown in Table 1. The energy transfer 
process consists of two parts in Mode 1: (a) the maximum voltage cell $\mathrm{B}_{i}$ discharges; (b) the outer-layer inductor $L_{i+2}$ charges. The two parts are presented in Figure 5.

Table 1. Switches working in Mode 1

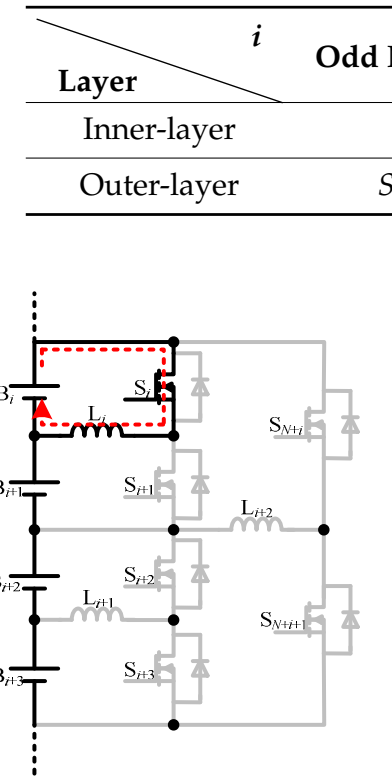

(a)

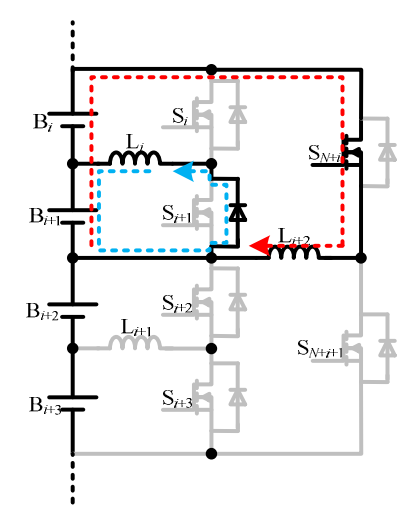

(b)

Figure 5. Current paths of second stage equalization in Mode 1. (a) The maximum voltage cell $\mathrm{B}_{i}$ discharges. (b) The out-layer inductor $\mathrm{L}_{i+2}$ charges.

As shown in Figure 5a, when the inner-layer switch $S_{i}$ is turned on, the analysis process is the same as the first stage equalization, the energy that stored in $L_{i}$ can be obtained from Formula (6). When $S_{i}$ is turned off, the inductor current $i_{L i}$ flows from the parasitic diode of $S_{i+1}$ to the adjacent cell $\mathrm{B}_{i+1}$. The equalizer unit transfers the excess energy from the highest voltage cell $\mathrm{B}_{i}$ to the adjacent cell $\mathrm{B}_{i+1}$. On the other hand, as shown in Figure $5 \mathrm{~b}$, when the outer-layer switches $\mathrm{S}_{N+i}$ is turned on, the cell $\mathrm{B}_{i+1}$ charges the outer-layer inductor $L_{i+2}$. The energy $W_{\mathrm{ri+1}}$ transfers from cell $\mathrm{B}_{i+1}$ to $L_{i+2}$ can be expressed as

$$
W_{r i+1}=V_{\mathrm{B} i+1} \int_{0}^{D_{n+i} T_{s}}\left(\frac{V_{\mathrm{B} i}+V_{\mathrm{B} i+1}}{2 L} t\right) d t=\frac{\left(V_{\mathrm{B} i}+V_{\mathrm{B} i+1}\right) V_{\mathrm{B} i+1}}{2 L} D_{N+i}^{2} T_{s}^{2}
$$

If the energy of cell $\mathrm{B}_{i+1}$ maintains dynamic balance in the process of charging and discharging, the process transfers excess energy from cell $\mathrm{B}_{i}$ to $L_{i+2}$, and the energy of cell $\mathrm{B}_{i+1}$ is unchanged. The corresponding energy relationship can be expressed as

$$
W_{i}=W_{r i+1}
$$

From Formula (11), the duty cycle $D_{N+i}$ of $S_{N+i}$ can be obtained as

$$
D_{N+i}=D_{i} V_{\mathrm{B} i} \sqrt{\frac{1}{\left(V_{\mathrm{B} i}+V_{\mathrm{B} i+1}\right) V_{\mathrm{B} i+1}}}
$$

In addition, the energy $W_{L i+2}$ transferred from the cell substring $\left(\mathrm{B}_{i}, \mathrm{~B}_{i+1}\right)$ to $L_{i+2}$ during this process can be expressed as

$$
W_{L i+2}=\left(V_{\mathrm{B} i}+V_{\mathrm{B} i+1}\right) \int_{0}^{D_{i+6} T_{s}} \frac{\left(V_{\mathrm{B} i}+V_{\mathrm{B} i+1}\right)}{L} t d t=\frac{\left(V_{\mathrm{B} i}+V_{\mathrm{B} i+1}\right)^{2}}{2 L} D_{N+i}^{2} T_{s}^{2}
$$


Mode 2: the energy flows among outer-layer inductors.

Whether switches work or not in equalization process is related to the position of maximum voltage cell $\mathrm{B}_{i}$ and minimum voltage cell $\mathrm{B}_{j}$ in Mode 2 , and the corresponding relationship is shown in Table 2. In addition, the energy transfer process consists of three parts in Mode 2: (a) the outer-layer inductor $L_{i+2}$ discharges; (b) the outer-layer inductor $L_{i+3}$ charges; (c) the outer-layer inductor $L_{j+1}$ charges. The three parts are presented in Figure 6.

Table 2. Switches working in Mode 2.

\begin{tabular}{ccc}
\hline$j$ & Odd Number & Even Number \\
\hline Odd number & $S_{N+i+2}, S_{N+i+4}, \ldots, S_{N+j-4}, S_{N+j-2}$ & $S_{N+i+1}, S_{N+i+3}, \ldots, S_{N+j-4}, S_{N+j-2}$ \\
\hline Even number & $S_{N+i+2}, S_{N+i+4}, \ldots, S_{N+j-3}, S_{N+j-1}$ & $S_{N+i+1}, S_{N+i+3}, \ldots, S_{N+j-3}, S_{N+j-1}$ \\
\hline
\end{tabular}

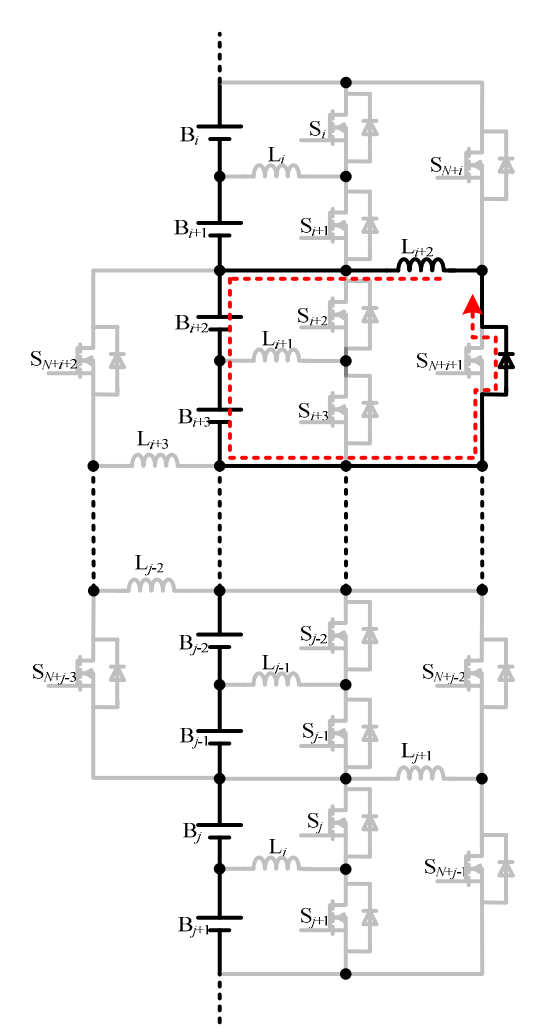

(a)

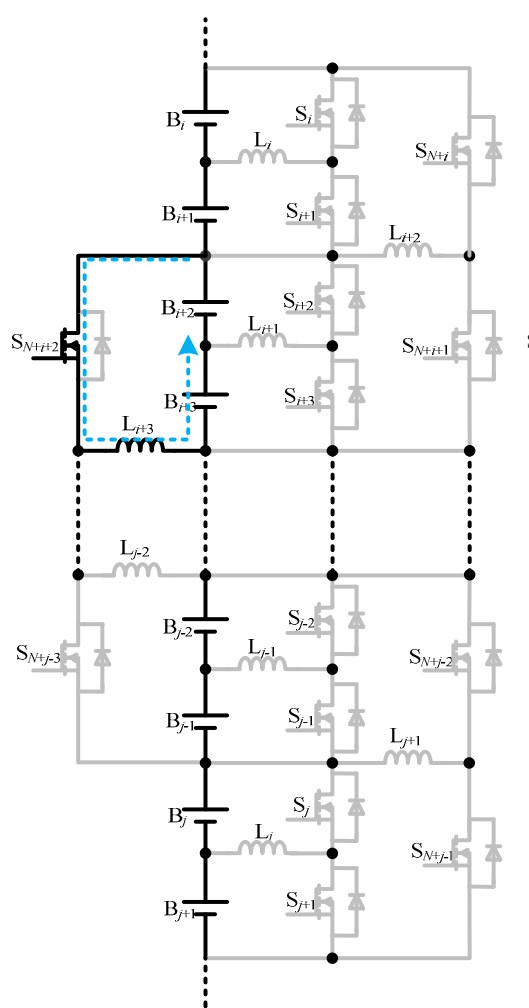

(b)

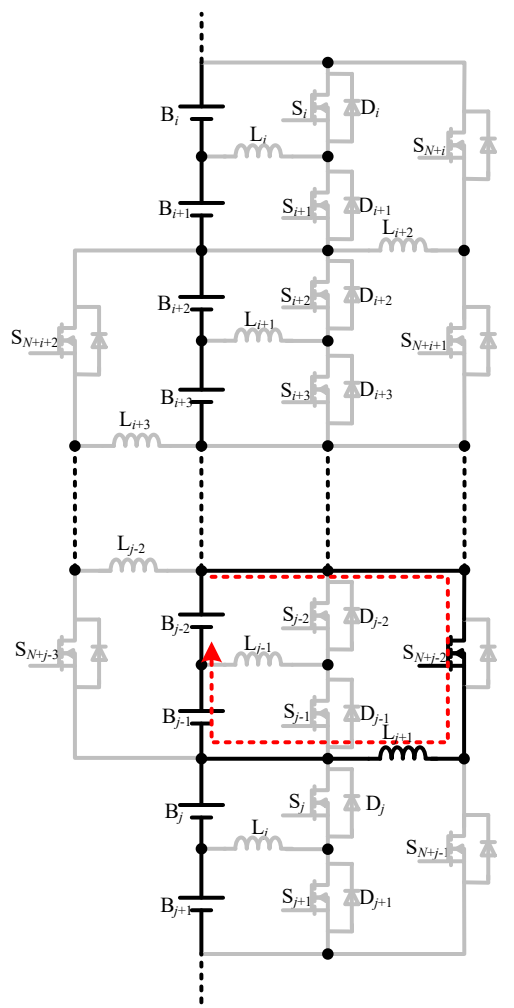

(c)

Figure 6. Current paths of the second stage equalization in Mode 2. (a) Outer-layer inductor $L_{i+2}$ discharges. (b) Outer-layer inductor $L_{i+3}$ charges. (c) Outer-layer inductor $L_{j+1}$ charges.

As shown in Figure 6a, when the switch $S_{N+i}$ is turned off, $L_{i+2}$ charges the adjacent cell substrings through the parasitic diode of switch $S_{N+i+1}$ to realize the energy transfer from $L_{i+2}$ to the cell substring $\left(\mathrm{B}_{i+2}, \mathrm{~B}_{i+3}\right)$. Then, as shown in Figure $6 \mathrm{~b}$, when the outer-layer switch $\mathrm{S}_{\mathrm{N}+i+2}$ is turned on, the cell substring $\left(\mathrm{B}_{i+2}, \mathrm{~B}_{i+3}\right)$ charges the outer-layer inductor $L_{i+3}$. In this process, the energy $W_{L i+3}$ transfers from the cell substring $\left(\mathrm{B}_{i+2}, \mathrm{~B}_{i+3}\right)$ to $L_{i+3}$, which can be expressed as

$$
W_{L i+3}=\left(V_{\mathrm{B} i+2}+V_{\mathrm{B} i+3}\right) \int_{0}^{D_{i+8} T_{s}} \frac{\left(V_{\mathrm{B} i+2}+V_{\mathrm{B} i+3}\right)}{L} t d t=\frac{\left(V_{\mathrm{B} i+2}+V_{\mathrm{B} i+3}\right)^{2}}{2 L} D_{N+i+2}^{2} T_{s}^{2}
$$


If the energy of cell substring $\left(\mathrm{B}_{i+2}, \mathrm{~B}_{i+3}\right)$ maintains dynamic balance in the process of charging and discharging, the mode transfers excess energy from cell $\mathrm{B}_{i}$ to $L_{i+3}$, and the energy of cell substring $\left(\mathrm{B}_{i+2}, \mathrm{~B}_{i+3}\right)$ is unchanged in this process. The corresponding energy relationship can be expressed as

$$
W_{L i+2}=W_{L i+3}
$$

From Formula (15), the duty cycle $D_{N+i+2}$ of $S_{N+i+2}$ can be expressed as

$$
D_{N+i+2}=\frac{V_{\mathrm{B} i}+V_{\mathrm{B} i+1}}{V_{\mathrm{B} i+2}+V_{\mathrm{B} i+3}} D_{N+i}
$$

Similarly, owing to the unchanged energy of intermediate cell substrings in this mode, it means that the duty cycle of the corresponding switches can be solved. Meanwhile, the energy that stored in the inductor $L_{j-2}$ can be obtained from Formula (6). When the outer-layer switch $S_{N+j-4}$ is turned off, the inductor $L_{j-2}$ charges the cell substring $\left(B_{j-2}, B_{j-1}\right)$ through the parasitic diode of switch $S_{N+j-3}$. Then, as shown in Figure $6 c$, when the switch $S_{N+j-2}$ is turned on, the cell substring $\left(B_{j-2}, B_{j-1}\right)$ charges the outer-layer inductor $L_{j+1}$. Similar to the above Formulas (13), (14), (15), duty cycle $D_{N+j-2}$ of $S_{N+j-2}$ can be expressed as

$$
D_{N+j-2}=\frac{V_{\mathrm{B} i}+V_{\mathrm{B} i+1}}{V_{\mathrm{B} j-2}+V_{\mathrm{B} j-1}} D_{N+i}
$$

Mode 3: the energy transfers from outer-layer inductor to the minimum voltage cell.

Whether switches work or not in equalization process is related to the position of maximum voltage cell $\mathrm{B}_{i}$ in Mode 3, and the corresponding relationship is shown in Table 3. In addition, the energy transfer process consists of two parts in Mode 3: (a) the outer-layer inductor $L_{j+1}$ discharges; (b) the minimum voltage cell $\mathrm{B}_{j}$ charges. The two parts are presented in Figure 7.

Table 3. Switches working in Mode 3

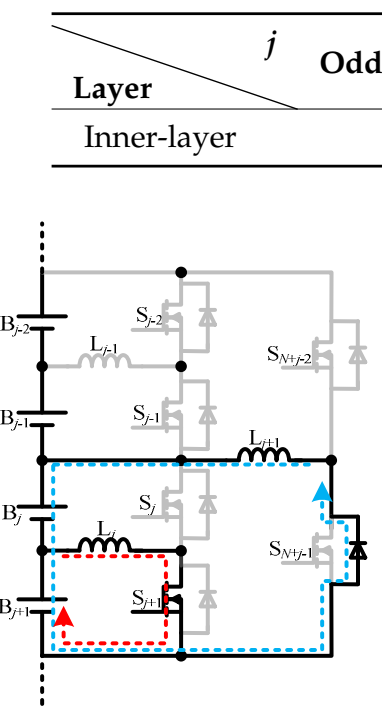

(a)

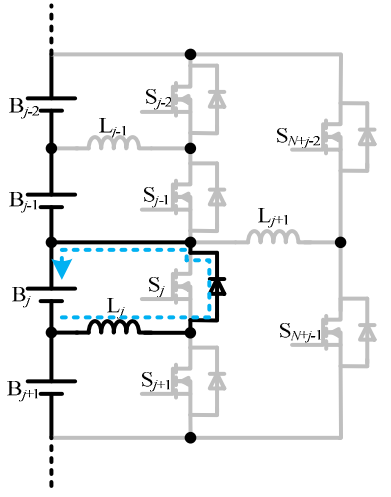

(b)

Figure 7. Current paths of the second stage equalization in Mode 3. (a) Outer-layer inductor $L_{j+1}$ discharges. (b) Minimum voltage cell $\mathrm{B}_{j}$ charges.

To simplify the analysis, it is assuming that all components is ideal, then the energy loss of the whole equalization process can be ignored. The energy transfers from the cell $\mathrm{B}_{i}$ to the inductor $L_{j+1}$ can be obtained from the Formula (13). As shown in Figure $7 \mathrm{a}$, when $S_{N+j-2}$ is turned off, the inductor current $i_{L j+1}$ commutated from $S_{N+j-2}$ to parasitic diode of outer-layer switch $S_{N+j-1}$, and the outer-layer 
equalizer unit transfers energy from $L_{j+1}$ to the cell substring $\left(\mathrm{B}_{j}, \mathrm{~B}_{j+1}\right)$. According to voltage-divider theorem, the energy $W_{s j+1}$ transferred from $L_{j+1}$ to the cell $B_{j+1}$ can be expressed as

$$
W_{s j+1}=\frac{V_{\mathrm{B} j+1}}{\left(V_{\mathrm{B} j}+V_{\mathrm{B} j+1}\right)} W_{L i+2}
$$

On the other hand, when the inner-layer switch $S_{j+1}$ is turned on, cell $\mathrm{B}_{j+1}$ charges the inner-layer inductor $L_{j}$. The energy $W_{\mathrm{r} j+1}$ transferred from the cell $\mathrm{B}_{j+1}$ to $L_{j}$ can be expressed as

$$
W_{r j+1}=V_{\mathrm{B} j+1} \int_{0}^{D_{j+1} T_{s}} \frac{V_{\mathrm{B} j+1}}{L} t d t=\frac{V_{\mathrm{B} j+1}^{2}}{2 L} D_{j+1}^{2} T_{s}^{2}
$$

If the energy of cell $\mathrm{B}_{j+1}$ maintains dynamic balance in the process of charging and discharging, the mode transfers excess energy from the maximum voltage cell $\mathrm{B}_{i}$ to $L_{j}$, and the energy of cell $\mathrm{B}_{j+1}$ is unchanged in this process. The corresponding energy relationship can be expressed as

$$
W_{s j+1}=W_{r j+1}
$$

From Formulas (18), (19), and (20), the duty cycle $D_{j+1}$ of $S_{j+1}$ can be expressed as

$$
D_{j+1}=D_{N+i}\left(V_{\mathrm{B} i}+V_{\mathrm{B} i+1}\right) \sqrt{\frac{1}{\left(V_{\mathrm{B} j}+V_{\mathrm{B} j+1}\right) V_{\mathrm{B} j+1}}}
$$

As shown in Figure $7 \mathrm{~b}$, when $S_{j+1}$ is turned off, $L_{j}$ charges the cell $\mathrm{B}_{j}$ through the parasitic diode of inner-layer switch $\mathrm{S}_{j}$ to realize the energy transfer from $L_{j}$ to cell $\mathrm{B}_{j}$.

In fact, the energy transfers from the maximum voltage cell $\mathrm{B}_{i}$ to the minimum voltage cell $\mathrm{B}_{j}$ by the second stage equalization, and the energy of other cells are unchanged. Thus, the second stage equalization decreases the maximum voltage gap between maximum voltage and minimum voltage in the battery string.

\section{Equalization Control Strategy}

The paper proposes a two stages equalization control strategy. The first stage equalization is widely used in traditional equalizers, it has the advantages of fast equalization speed and simply control, but it has the disadvantage of repeated flow of energy. Meanwhile, the maximum voltage gap after balancing among cells is increased with the increase of $N$ due to the accumulation of threshold voltage gap of equalizers. Especially for AC2C equalizer, if the voltage gap of two adjacent cells is less than the predefined threshold, then in the worst case, the maximum voltage gap between maximum voltage and minimum voltage in the battery string can be expressed as

$$
V_{\text {gap }}=(N-1) \Delta V
$$

The increase of maximum voltage gap after balancing among cells affects the equalization performance, which will decrease the total storage capacity and battery lifecycle. Meanwhile, if the given threshold is reduced, although the maximum voltage gap will also decrease, the corresponding equalization time will be greatly increased. Therefore, the second stage equalization is proposed to decrease the maximum voltage gap.

The flow chart of the proposed equalization control strategy used in simulations and experiments is show in Figure 8. When the arbitrary equalizer unit detects that two adjacent cells or cell substrings voltage gap is greater than the predefined threshold, the first stage equalization starts working. On the contrary, when all equalization units detect that the adjacent voltage gap is less than the given threshold, the first stage equalization stops working. Unfortunately, the maximum voltage gap is still large after 
finishing the first stage equalization, the equalizer begins the second stage equalization, and it stops working until the maximum voltage gap is less than the predefined threshold. Thus, the proposed control strategy can improve equalization performance.

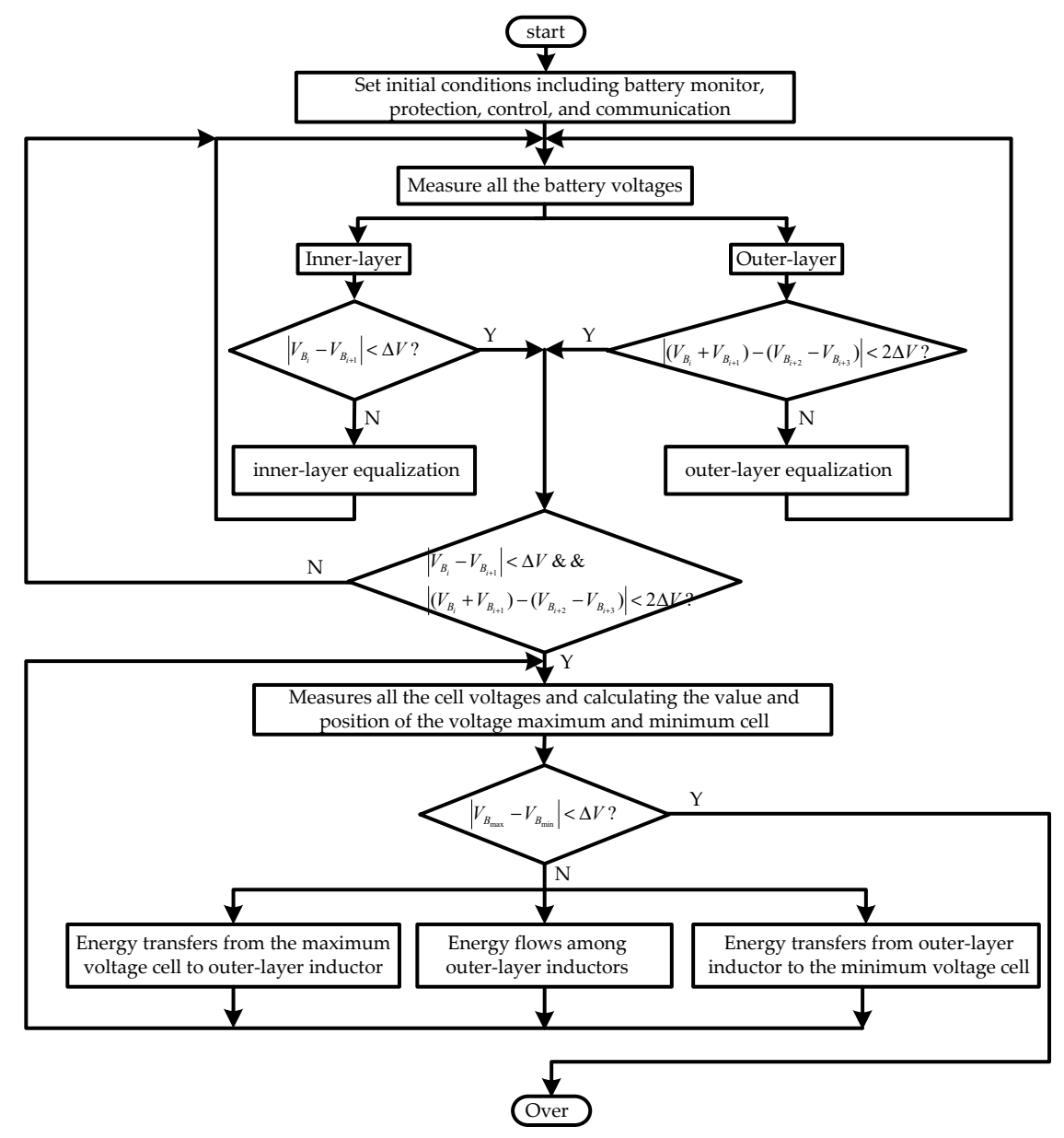

Figure 8. Flow chart of proposed equalization control strategy used in simulations and experiments.

\section{Simulation}

In order to compare the equalization performance of proposed equalizer with $A C 2 C$ equalizer, the simulations for six cells at six different initial voltage distributions were carried out using PSIM software. Table 4 shows the initial voltage distributions of six cells.

Table 4. Initial voltage distributions for simulation

\begin{tabular}{ccccccc}
\hline & $\mathbf{B}_{\mathbf{1}}(\mathbf{V})$ & $\mathbf{B}_{\mathbf{2}}(\mathbf{V})$ & $\mathbf{B}_{\mathbf{3}}(\mathbf{V})$ & $\mathbf{B}_{\mathbf{4}}(\mathbf{V})$ & $\mathbf{B}_{\mathbf{5}}(\mathbf{V})$ & $\mathbf{B}_{\mathbf{6}}(\mathbf{V})$ \\
\hline Case 1 & 3.21 & 3.47 & 3.35 & 3.72 & 3.13 & 3.64 \\
\hline Case 2 & 3.21 & 3.47 & 3.13 & 3.64 & 3.35 & 3.72 \\
\hline Case 3 & 3.35 & 3.72 & 3.21 & 3.47 & 3.13 & 3.64 \\
\hline Case 4 & 3.35 & 3.72 & 3.13 & 3.64 & 3.21 & 3.47 \\
\hline Case 5 & 3.13 & 3.64 & 3.21 & 3.47 & 3.35 & 3.72 \\
\hline Case 6 & 3.13 & 3.64 & 3.35 & 3.72 & 3.21 & 3.47 \\
\hline
\end{tabular}

As shown in Table 4, at different cases, the maximum voltage gaps among cells are $0.59 \mathrm{~V}$. In simulation, the switching frequency was set as $10 \mathrm{kHz}$, and the value of each inductor is $100 \mu \mathrm{H}$ 
and duty cycle was set as 0.4 . In addition, energy storage cells $B_{1}-B_{6}$ were replaced by capacitors (0.1F). The paper compares three equalizers by simulation: AC2C equalizer, DLE equalizer with first stage equalization (DLE1 equalizer), and DLE equalizer with the proposed two-stage equalization (DLE2 equalizer). For the AC2C equalizer, simulation and experimental voltage thresholds are set to $10 \mathrm{mV}$. For DLE1 equalizer, the inner-layer voltage threshold was set as $10 \mathrm{mV}$ and outer-layer voltage threshold was set as $20 \mathrm{mV}$. Furthermore, for DLE2 equalizer, the maximum voltage gap of the whole battery pack was set as $10 \mathrm{mV}$.

The three equalizers are simulated using identical voltage distribution and parameters. Under the condition of six cells, the number of different initial voltage distribution sequences is $A_{6}^{6}=720$. It is almost impossible to simulate those distributions one by one. However, for DLE equalizer, six cells can be divided into three substrings that include two adjacent cells, so the paper compares six different voltage distributions due to $A_{3}^{3}=6$.

The simulation results are shown in Figure 9. From the simulation results, at six different initial voltage distributions, DLE2 equalizer has controllable maximum voltage gap compared to the other equalizers. Furthermore, the DLE equalizer has a relatively stable equalization time than AC2C equalizer in different distribution sequences of the cell voltage, it means that DLE equalizer has more stable equalization performance.

The paper also defines voltage variance and maximum voltage gap as extra index for further evaluate the equalization performance.

The variance $\sigma^{2}$ is defined as

$$
\sigma^{2}=\frac{1}{N} \sum_{i=1}^{n}\left(V_{\mathrm{B} i}-\bar{V}\right)^{2}
$$

where $\bar{V}$ is the average voltage of cells in the battery string. The smaller $\sigma^{2}$ is, the better equalization performance is.

The maximum voltage gap $V_{g a p}$ in the battery string is defined as

$$
V_{\text {gap }}=V_{B \max }-V_{B \min }
$$

where $V_{B \max }$ is maximum voltage in the battery string and $V_{B \min }$ is minimum voltage in the battery string. Similarly, the smaller $V_{g a p}$ is, the better equalization performance is.

Table 5 shows the average equalization performance of six simulation results. The DLE1 and DLE2 equalizers have shorter average equalization time, smaller voltage variance, and smaller maximum voltage gap compare with AC2C equalizer, which further proves that DLE1 and DLE2 equalizers have superior equalization performance. As $N$ grows, the advantages of DLE1 and DLE2 equalizers will become more and more obvious. In addition, although the average equalization time of DLE2 equalizer increases slightly compared with DLE1 equalizer, the voltage variance and the maximum voltage gap is significant declined.

Table 5. Average equalization performance of six simulation results

\begin{tabular}{cccc}
\hline & AC2C & DLE1 & DLE2 \\
\hline Equalization time $(\mathrm{ms})$ & 90.67 & 85.42 & 88.97 \\
Variance & $2.3 \times 10^{-4}$ & $8.16 \times 10^{-5}$ & $2.17 \times 10^{-5}$ \\
Voltage gap (V) & 0.0421 & 0.0267 & 0.0097 \\
\hline
\end{tabular}



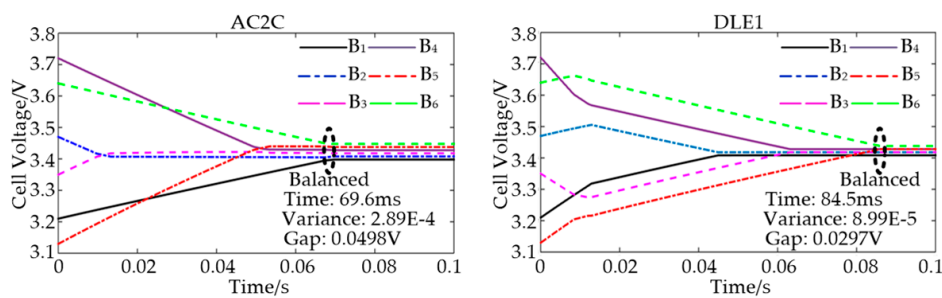

(a)
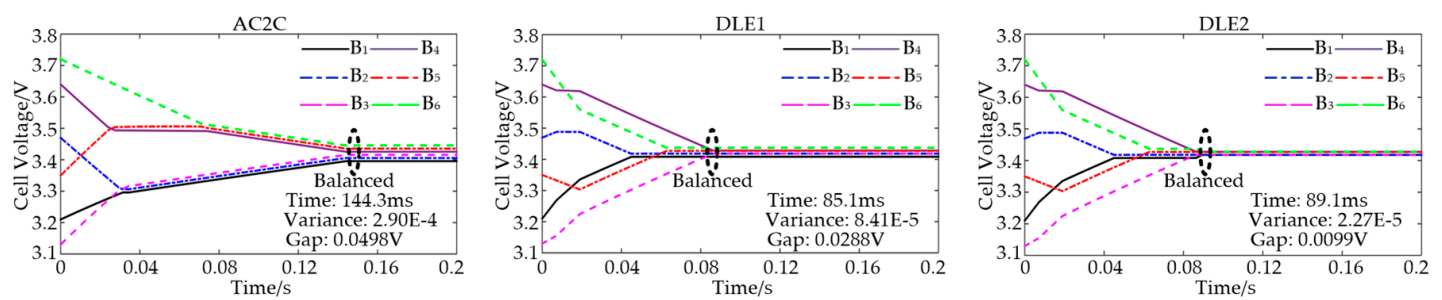

(b)
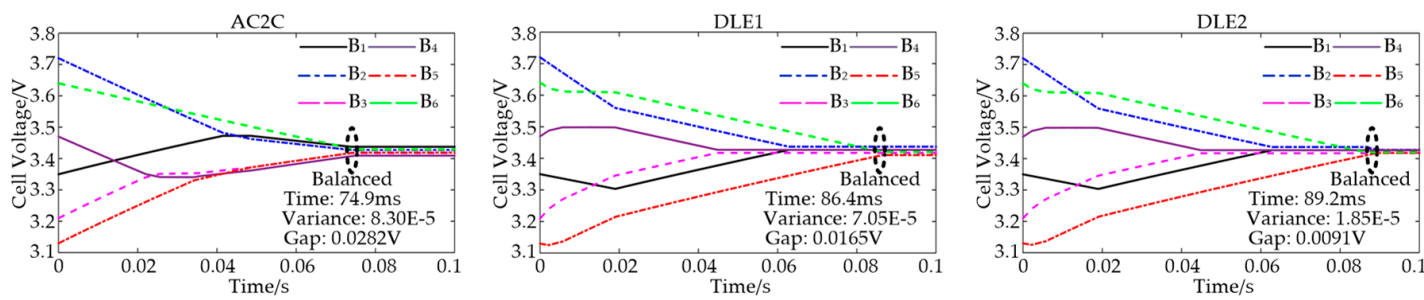

(c)
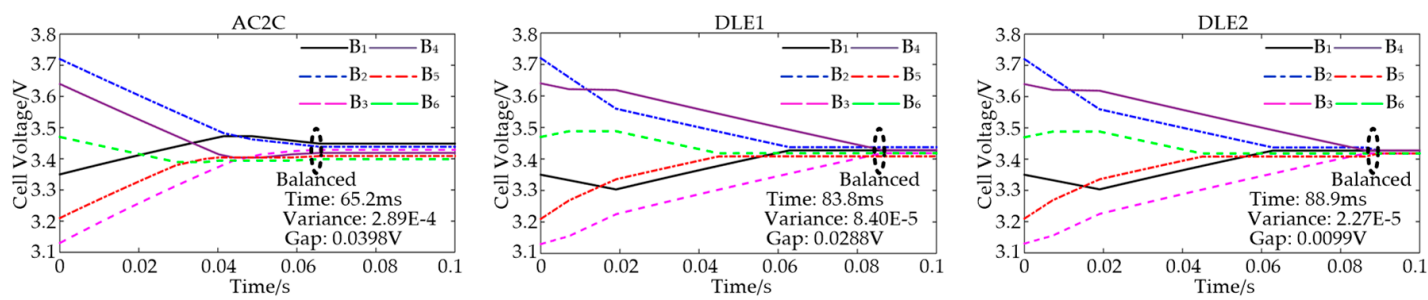

(d)
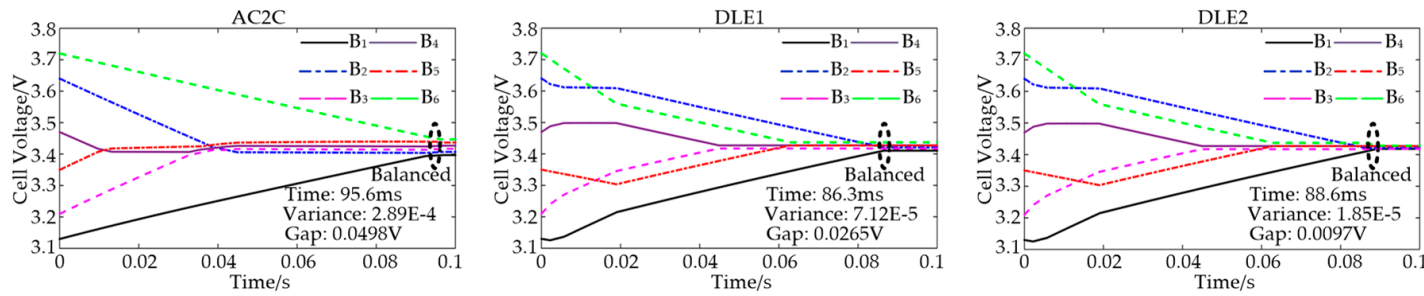

(e)
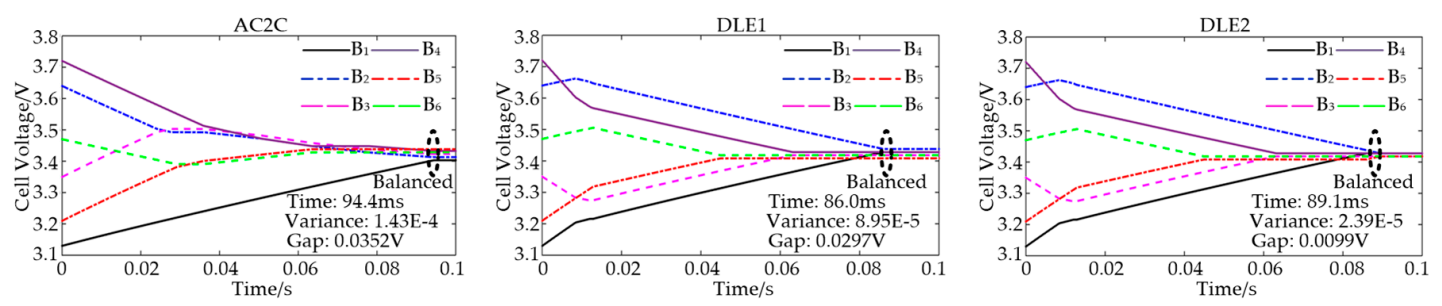

(f)

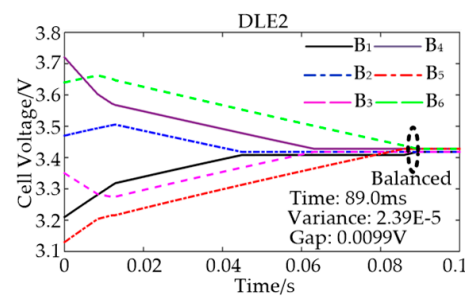




\section{Experimental Results}

In order to verify the analysis and simulation results in above sections, experiments were carried out by using lithium-ion battery string containing six cells connected in series. Figure 10 shows a photograph of the experimental circuit. The acquisition chip is the sensor to make measurement on the status of the battery cell. The monitoring IC reads the voltage value of the cells and communication with the microcontroller. The microcontroller collects data and sends it to upper computer. The microcontroller comparers the result with the threshold value set, detects the unbalanced cell and generates pulse width modulation (PWM) signals to drive the corresponding MOSFET according to the proposed algorithm. Figure 10 shows a photograph of the experimental circuit and Table 6 shows the specification of component used for experiments.

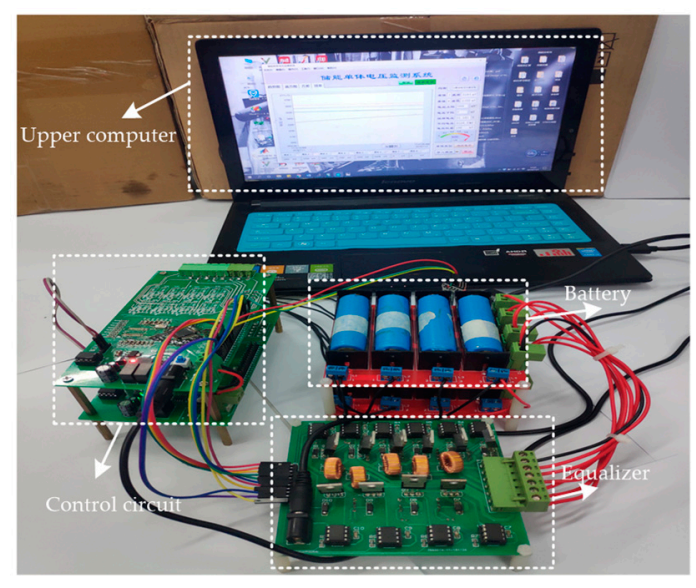

Figure 10. Photograph of the experimental circuit.

Table 6. Specification of component for experiments

\begin{tabular}{ccc}
\hline \multicolumn{2}{c}{ Parameters } & Value \\
\hline & MOSFET & IRF540NPBF \\
& Gate driver & HCPL-3120 \\
Cell balancing circuit & Switching frequency & $10 \mathrm{KHz}$ \\
& Inductor & $100 \mu \mathrm{H}$ \\
& Microcontroller & STM32F103RCT6 \\
& Acquisition chip & LTC6803 \\
\hline \multirow{2}{*}{ Lithium-ion battery } & Nominal capacity & $2 \mathrm{Ah}$ \\
& Cell voltage variation & $2.8-4.2 \mathrm{~V}$ \\
\hline
\end{tabular}

Figure 11 shows the experimental results for six cells under the condition of Case 2 of Table 4 . The operating conditions of $\mathrm{AC} 2 \mathrm{C}$ equalizer and the proposed equalizers are the same as the simulation. As shown in Figure 11, three equalizers can realize the equalization of battery string, but their balancing time is different. The AC2C equalizer takes $440 \mathrm{~min}$. However, the proposed equalizers require only $280 \mathrm{~min}$ and $290 \mathrm{~min}$. Thus, the equalization speed of the proposed equalizers is better than that of the conventional $\mathrm{AC} 2 \mathrm{C}$ equalizer. In addition, the maximum voltage gap of conventional $\mathrm{AC} 2 \mathrm{C}$ equalizer is $0.045 \mathrm{~V}$ after the total equalization process. The maximum voltage gap of DLE1 equalizer is $0.025 \mathrm{~V}$ after balancing, and it is reduced by $55.6 \%$ compared with $\mathrm{AC} 2 \mathrm{C}$ equalizer. For the DLE2 equalizer, although the equalization time equalizer increases slightly compared with DLE1 equalizer, the maximum voltage gap is reduced from $0.025 \mathrm{~V}$ to $0.009 \mathrm{~V}$, which is the minimum among three equalizers. Meanwhile, the voltage variance of the proposed equalizers is smaller than the AC2C equalizer. 


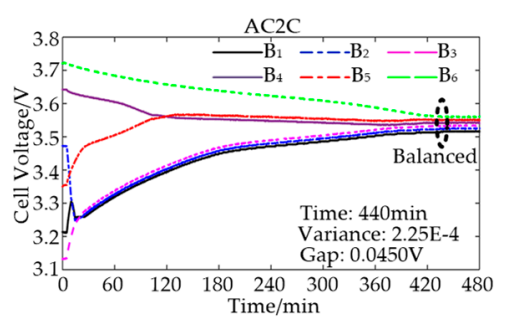

(a)

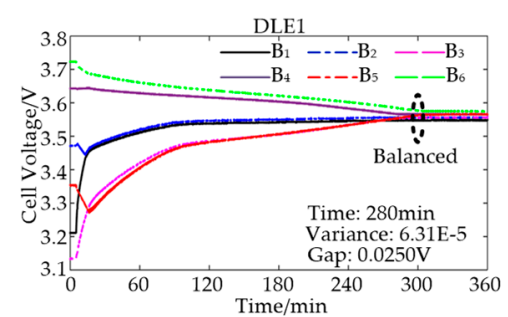

(b)

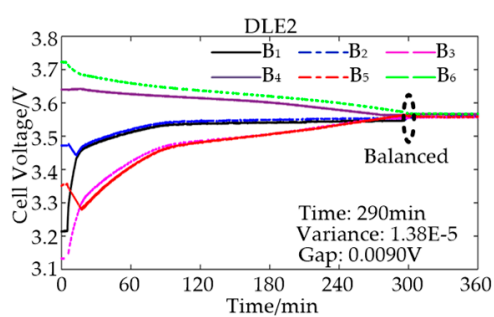

(c)

Figure 11. Experimental results of three equalizers for six cells under the condition of Case 2 of Table 4 . (a) AC2C; (b) DLE1; (c) DLE2.

Figure 12 shows the cell voltage distributions of three equalizers after balancing. Among them, the maximum voltage gap of the DLE2 equalizer is minimum, followed by DLE1 equalizer, and the maximum voltage gap of the $\mathrm{AC} 2 \mathrm{C}$ equalizer is maximum. Thus, it is further demonstrated that the proposed equalizers can achieve better equalization performance.

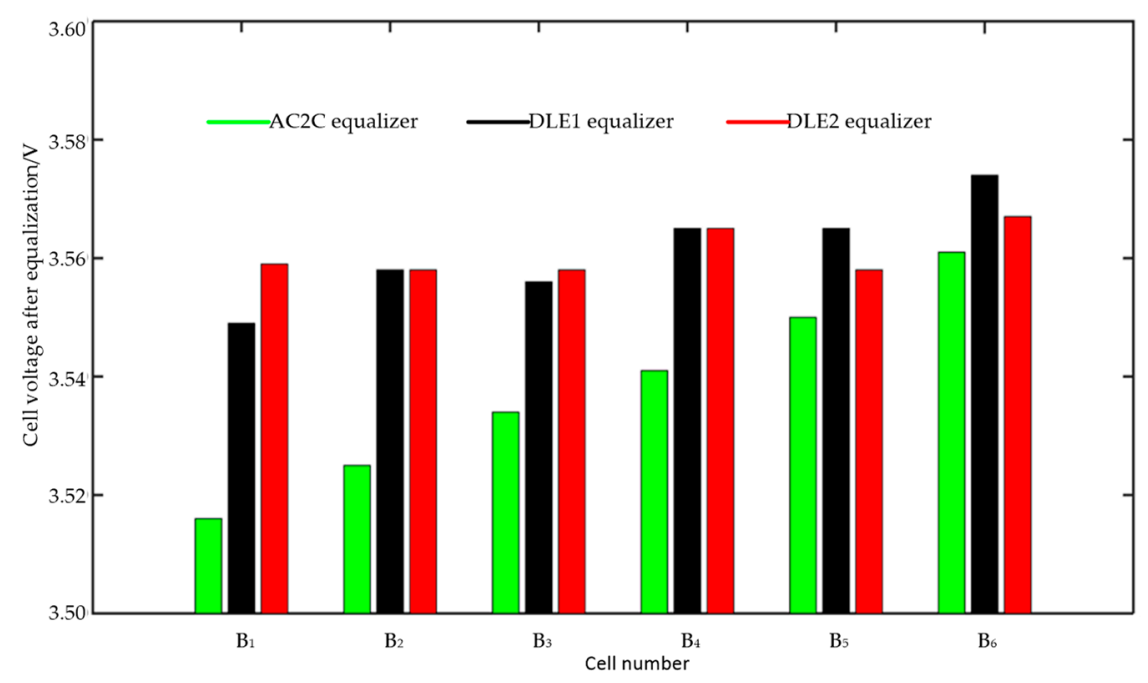

Figure 12. Cell voltage distributions of three equalizers after balancing.

In summary, DLE1 and DLE2 equalizers realize better equalization performance compared with $\mathrm{AC} 2 \mathrm{C}$ equalizer. In addition, the DLE2 equalizer decreases efficiency and speed a little compared with the DLE1 equalizer due to adding second stage equalization, but the maximum voltage gap and variance are minimum of the DLE2 equalizer among three equalizers. Furthermore, the maximum voltage gap of the DLE2 equalizer is controllable and does not increase as $N$ increases. It means that the DLE2 equalizer can better prolong battery lifecycle and available capacity compared with the AC2C equalizer and the DLE1 equalizer. However, as in any engineering problem, the DLE2 equalizer exists as a tradeoff. The DLE2 equalizer increases equalization performance, but it also increases complexity of the control strategy compared with the AC2C equalizer and DLE1 equalizer.

\section{Conclusions}

This paper proposes a new double-layer E-structure (DLE) equalizer for battery voltage equalization, in which the equalization time can be decreased compared with $A C 2 C$ equalizer. In addition, a novel two-stage control strategy is proposed to decrease the maximum voltage gap. The novel control strategy is very useful to meet higher cell voltage consistency. Furthermore, simulations and experiments are used to verify the validity of the proposed equalizer and control strategy. Thus, it is anticipated that the proposed equalizer and control strategy will allow important improvements in the performance of energy storage systems. 
Author Contributions: This research article has four authors. The circuit structure and control strategy were designed by S.X. and K.G. X.Z., K.L. and K.G. conceived the research methods. S.X. contributed the experimental environment. K.G. wrote the paper. X.Z. and S.X. revised the paper and provided some helpful suggestions.

Funding: This research was supported by Sichuan Science and Technology Program under Grants (61371033). The authors would like to thank them for their support and help. The authors would also like to thank the reviewers for their corrections and helpful suggestions.

Conflicts of Interest: The authors declare no conflict of interest.

\section{References}

1. Cassani, P.; Williamson, S. Significance of battery cell equalization and monitoring for practical commercialization of plug-in hybrid electric vehicles. In Proceedings of the 2009 Twenty-Fourth Annual IEEE Applied Power Electronics Conference and Exposition, Washington, DC, USA, 15-19 February 2009; pp. 4654-4671.

2. Chan, C.; Chau, K. An overview of electric vehicles-challenges and opportunities. In Proceedings of the 22nd International Conference on Industrial Electronics, Control, and Instrumentation, Taipei, Taiwan, 9 August 1996; pp. 1-6.

3. Cao, J.; Schofield, N.; Emadi, A. Battery balancing methods: A comprehensive review. In Proceedings of the 2008 IEEE Vehicle Power and Propulsion Conference, Harbin, China, 3-5 September 2008; pp. 1-6.

4. Linzen, D.; Buller, S.; Karden, E.; Doncker, R.W.D. Analysis and evaluation of charge balancing circuits on performance, reliability and lifetime of supercapacitor systems. IEEE Trans. Ind. Appl. 2005, 41, 1135-1141. [CrossRef]

5. Daowd, M.; Omar, N.; Bossche, P.V.D.; Mierlo, J.V. A review of passive and active battery balancing based on Matlab/Simulink. In Proceedings of the 2011 IEEE Vehicle Power and Propulsion Conference, Chicago, IL, USA, 6-9 September 2011; pp. 2974-2989.

6. Lindemark, B. Individual cell voltage equalizers (ICE) for reliable battery performance. In Proceedings of the Thirteenth International Telecommunications Energy Conference-INTELEC 91, Kyoto, Japan, 5-8 November 1991; pp. 196-201.

7. Pascual, C.; Krein, P. Switched capacitor system for automatic series battery equalization. In Proceedings of the Applied Power Electronics Conference, Atlanta, GA, USA, 27 February 1997; pp. 848-854.

8. Ye, Y.; Cheng, K.W.E. An automatic switched-capacitor cell balancing circuit for series-connected battery string. Energies 2016, 9, 138. [CrossRef]

9. Kim, M.; Kim, C.; Kim, J.; Moon, G. A chain structure of switched capacitor for improved cell balancing speed of lithium-ion batteries. IEEE Trans. Ind. Electron. 2014, 61, 3989-3999. [CrossRef]

10. Kutkut, N.; Wiegman, H.; Divan, D.; Novotny, D. Design considerations for charge equalization of an electric vehicle battery system. IEEE Trans. Ind. Appl. 1999, 35, 28-35. [CrossRef]

11. Cassani, P.A.; Williamson, S.S. Design, testing, and validation of a simplified control scheme for a novel plug-in hybrid electric vehicle battery cell equalizer. IEEE Trans. Ind. Electron. 2010, 57, 3956-3962. [CrossRef]

12. Guo, X.; Kang, L.; Huang, Z.; Yao, Y.; Yang, H. Research on a novel power inductor-based bidirectional lossless equalization circuit for series-connected battery packs. Energies 2015, 8, 5555-5576. [CrossRef]

13. Wang, S.; Kang, L.; Guo, X.; Wang, Z.; Liu, M. A novel layered bidirectional equalizer based on a buck-boost converter for series-connected battery strings. Energies 2017, 10, 1011. [CrossRef]

14. Peng, F.; Wang, H.; Yu, L. Analysis and design considerations of efficiency enhanced hierarchical battery equalizer based on bipolar CCM buck-boost unit. IEEE Trans. Ind. Appl. 2019, 55, 4053-4063. [CrossRef]

15. Kim, M.; Kim, J.; Moon, G. Center-cell concentration structure of a cell-to-cell balancing circuit with a reduced number of switches. IEEE Trans. Power Electron. 2014, 29, 5285-5297. [CrossRef]

16. Dong, B.; Li, Y.; Han, Y. Parallel architecture for battery charge equalization. IEEE Trans. Power Electron. 2015, 30, 4906-4913. [CrossRef]

17. Lee, K.; Lee, S.; Choi, Y.; Kang, B. Active balancing of Li-Ion battery cells using transformer as energy carrier. IEEE Trans. Ind. Electron. 2017, 64, 1251-1257. [CrossRef] 
18. Park, H.; Kim, J.; Kim, C.; Moon., G.; Lee, J. A modularized charge Equalizer for an HEV lithium-ion battery string. IEEE Trans. Ind. Electron. 2009, 56, 1464-1476. [CrossRef]

19. Uno, M.; Kukita, A. String-to-battery voltage equalizer based on a half-bridge converter with multistacked current doublers for series-connected batteries. IEEE Trans. Power Electron. 2019, 34, 1286-1298. [CrossRef]

(C) 2019 by the authors. Licensee MDPI, Basel, Switzerland. This article is an open access article distributed under the terms and conditions of the Creative Commons Attribution (CC BY) license (http://creativecommons.org/licenses/by/4.0/). 